\title{
Indifferentiability of Permutation-Based Compression Functions and Tree-Based Modes of Operation, with Applications to MD6
}

\author{
Yevgeniy Dodis ${ }^{1}$, Leonid Reyzin ${ }^{2}$, Ronald L. Rivest ${ }^{3}$, and Emily Shen ${ }^{3}$ \\ 1 New York University \\ dodis@cs.nyu.edu \\ 2 Boston University \\ reyzin@cs.bu.edu \\ 3 Massachusetts Institute of Technology \\ $\{$ rivest, eshen\}@csail.mit.edu
}

\begin{abstract}
MD6 [17] is one of the earliest announced SHA-3 candidates, presented by Rivest at CRYPTO'08 [16]. Since then, MD6 has received a fair share of attention and has resisted several initial cryptanalytic attempts 111.

Given the interest in MD6, it is important to formally verify the soundness of its design from a theoretical standpoint. In this paper, we do so in two ways: once for the MD6 compression function and once for the MD6 mode of operation. Both proofs are based on the indifferentiability framework of Maurer et al. 13] (also see 9]).

The first proof demonstrates that the "prepend/map/chop" manner in which the MD6 compression function is constructed yields a compression function that is indifferentiable from a fixed-input-length (FIL), fixedoutput-length random oracle.

The second proof demonstrates that the tree-based manner in which the MD6 mode of operation is defined yields a hash function that is indifferentiable from a variable-input-length (VIL), fixed-output-length random oracle.

Both proofs are rather general and apply not only to MD6 but also to other sufficiently similar hash functions.

These results may be interpreted as saying that the MD6 design has no structural flaws that make its input/output behavior clearly distinguishable from that of a VIL random oracle, even for an adversary who has access to inner components of the hash function. It follows that, under plausible assumptions about those inner components, the MD6 hash function may be safely plugged into any application proven secure assuming a monolithic VIL random oracle.
\end{abstract}

\section{Introduction}

In light of recent devastating attacks on existing hash functions, such as MD4 [1822, MD5 [20, SHA-0 217, and SHA-1 [19, NIST recently announced a competition for a new hash function standard, to be called SHA-3 [14]. NIST

O. Dunkelman (Ed.): FSE 2009, LNCS 5665, pp. 104-121, 2009.

(C) International Association for Cryptologic Research 2009 
received 64 submissions, one of which is MD6. The current status of the SHA-3 competition can be found on the NIST web site1.

Crutchfield 10 showed that MD6 has many attractive properties required of a good hash function, such as preservation of collision-resistance, unpredictability, preimage-resistance, and pseudorandomness. As observed by Coron et al. 9], however, the above "traditional" properties of hash functions are often insufficient for many applications, which require that the hash function behaves "like a random oracle." Moreover, the initial NIST announcement for the SHA-3 competition [15] states that the candidate submissions will be judged in part by

"The extent to which the algorithm output is indistinguishable from a random oracle."

Thus, it is important to show that the design of the hash function is "consistent" with the proofs of security in the random oracle (RO) model [3. Such a notion of consistency with the random oracle model was recently defined by Coron et al. [9]; it is called indifferentiability from a random oracle and is based on the general indifferentiability framework of Maurer et al. [13].

Given the importance of the random oracle model in the design of practical cryptographic schemes and the increased popularity of the indifferentiability framework in the analysis of hash functions [92812 4], we suggest that it is critical that the winner of the SHA-3 competition satisfy such an "indifferentiability from a random oracle" property.

The main result of this paper is a formal proof that the design of MD6, both at the compression function level and at the mode of operation level, provides indifferentiability from a random oracle. Thus, the MD6 mode of operation and compression function have no structural flaws that would allow them to be distinguished from (VIL or FIL, respectively) random oracles. It follows, from results due to Maurer et al. 13, that, given reasonable assumptions about the permutation inside the compression function, the MD6 hash function may be safely plugged into any higher-level application whose security is proven assuming the hash function is a VIL random oracle.

These results generalize to other hash functions built on permutation-based compression functions and tree-based modes of operations, if they are sufficiently similar to MD6 in structure to meet the conditions of our proofs. We note that similar results on tree-based hashing have been obtained in independent work by Bertoni et al. [6].

To explain our results more precisely, we briefly recall the indifferentiability framework and the high level design of the MD6 hash function.

\subsection{Indifferentiability}

The notion of indifferentiability was first introduced by Maurer et al. in [13]. Informally, it gives sufficient conditions under which a primitive $F$ can be "safely replaced" by some construction $C^{G}$ (using an ideal primitive $G$ ). The formal definition is as follows.

${ }^{1}$ http://csrc.nist.gov/groups/ST/hash/sha-3/index.html 
Definition 1. A Turing machine $C$ with oracle access to an ideal primitive $G$ is $\left(t, q_{F}, q_{S}, \epsilon\right)$-indifferentiable from an ideal primitive $F$ if there exists a simulator $S$ such that, for any distinguisher $D$, it holds that:

$$
\left|\operatorname{Pr}\left[D^{C, G}=1\right]-\operatorname{Pr}\left[D^{F, S}=1\right]\right|<\epsilon
$$

The simulator $S$ has oracle access to $F$ (but does not see the queries of the distinguisher $D$ to $F$ ) and runs in time at most $t$. The distinguisher makes at most $q_{F}$ queries to $C$ or $F$ and at most $q_{S}$ queries to $G$ or $S$.

Indifferentiability is a powerful notion; Maurer et al. 13] show that if $C^{G}$ is indifferentiable from $F$, then $F$ may be replaced by $C^{G}$ in any cryptosystem, and the resulting cryptosystem is at least as secure in the $G$ model as in the $F$ model.

In this paper, $F$ will always be a random oracle - either fixed-input-length, fixed-output-length or variable-input-length, fixed-output-length. Thus, we will be showing that a certain construction $C$ is indifferentiable from a random oracle, meaning that any cryptosystem proven secure in the RO model will still be secure in the $G$ model, when the hash function is implemented using $C^{G}$.

\subsection{The MD6 Hash Function: High-Level View}

The MD6 function consists of the following two high-level steps. First, there is a compression function which operates on fixed-length inputs. Second, there is a mode of operation, which uses the compression functions as a black-box and to evaluate the hash function on arbitrary-length inputs. Naturally, our indifferentiability results will consist of two parts as well: (a) that the compression function, under some natural assumptions, is indifferentiable from a fixed-input-length RO, and (b) that, assuming the compression function is a fixed-input-length RO, the MD6 mode of operation yields a hash function that is indifferentiable from a variable-input-length $\mathrm{RO}$.

The Compression Function. The MD6 compression function $f$ maps an input $N$ of length $n=89$ words (consisting of 25 words of auxiliary input followed by 64 words of data) to an output of $c=16$ words. Thus, the compression function reduces the length of its data input by a factor of 4 . The compression function $f$ is computed as a series of operations, which we view as the application of a random permutation $\pi$ over the set of 89-word strings, followed by a truncation operation which returns the last 16 words of $\pi(N)$.

In each call to $f$ in an MD6 computation, the first 15 words of auxiliary input are a constant $Q$ (a representation of the fractional part of $\sqrt{6}$ ). Therefore, in our analysis, we consider the "reduced" compression function $f_{Q}$, where $f_{Q}(x)=$ $f(Q \| x)$. For the full specification of the MD6 compression function, we refer the reader to [17.

In Section 3, we prove that $f_{Q}$ is indifferentiable from a random oracle $F$, assuming that the main operation of the compression function is the application of a fixed public random permutation $\pi$. 
The Mode of Operation. The standard MD6 mode of operation is a hierarchical, tree-based construction to allow for parallelism. However, for devices with limited storage, the MD6 mode of operation can be iterative. There is an optional level parameter $L$ which allows a smooth transition between the fully hierarchial mode of operation and the fully iterative mode of operation. At each node in the tree, the input to the compression function includes auxiliary information. The auxiliary information includes a unique identifier $U$ for each node (consisting of a tree level and index). In addition, there is a bit $z$ which equals 1 in the input to the root node (the final compression call) and 0 in the input to all other nodes. For the full specification of the MD6 mode of operation, we refer the reader to 17 .

In Section 4, we prove that the MD6 mode of operation is indifferentiable from a random oracle $F$ when the compression function $f_{Q}$ is modeled as a random oracle. In fact, our proof is quite general and applies essentially to any tree-like construction, as long as the final computation node has a distinguishable input structure and compression function inputs are uniquely parsable into blocks that are either raw message bits, metadata, or outputs of the compression function on "child" nodes.

\section{Notation}

We first introduce some notation.

Let $\mathbf{W}=\{0,1\}^{w}$ denote the set of all $w=64$-bit words. Let $\chi_{a}(X)$ denote a function that returns the last $a$ bits of $X$.

\section{Indifferentiability from Random Oracle of MD6 Compression Function}

In this section, we will prove the indifferentiability from a random oracle of the MD6 compression function construction, under certain assumptions. The compression function construction involves three steps:

- Prepending a constant value $Q$ to the compression function input.

- Mapping the result by applying a fixed (pseudo)-random permutation $\pi$ to it, and

- Chopping (removing) bits off the front of the result, so that what remains has the desired length as a compression function output.

Our proof applies in general to compression functions constructed in this manner. Our presentation of the proof will use notation shared with the formal specification of MD6, for convenience.

We view the compression function as based on a fixed public random permutation $\pi(\cdot)$, i.e., $f(N)=\chi_{c w}(\pi(N))$. (Recall that $\chi_{c w}(\cdot)$ returns the last $c w$ bits of its input.) Since the permutation is public, an adversary can compute both $\pi$ and $\pi^{-1}$ easily. Therefore, we need to consider an adversarial model where the adversary has these powers. 
Note that in this model the adversary can both invert $f$ and find collisions for $f$ easily, if we do not do something additional. (This is because the adversary can take the $c$-word output $C$, prepend $n-c$ words of random junk, then apply $\pi^{-1}$ to get a valid pre-image for $C$. He can do this twice, with different junk values, to get a collision.) However, MD6 does have an important additional feature: a valid compression function input must begin with a fixed constant $Q$. We now proceed to show that this yields a compression function that behaves like a random oracle when $\pi$ is a random permutation.

Recall that $\mathbf{W}=\{0,1\}^{w}$ denotes the set of all $w$-bit words and that $f$ takes $n$-word inputs. We let $f_{Q}(x)=f(Q \| x)$ denotes the "reduced" compression function that takes $(n-q)$-word inputs, prepends the fixed prefix $Q$, and runs $f$. To make it explicit that in this section we are modeling $f$ and $f_{Q}$ in terms of a random permutation $\pi$ on $\mathbf{W}^{n}$, we will write

$$
f_{Q}^{\pi}(x)=\chi_{c w}(\pi(Q \| x)),
$$

where $\chi_{c w}(y)$ returns the last $c w$ bits of $y$, and where $x$ is in $\mathbf{W}^{n-q}$.

Let the ideal functionality be represented by $F: \mathbf{W}^{n-q} \rightarrow \mathbf{W}^{c}$, a random oracle with same signature as $f_{Q}^{\pi}$. We will show that $f_{Q}^{\pi}$ is indifferentiable from $F$, as stated below.

Theorem 1. If $\pi$ is a random permutation and $Q$ is arbitrary, the reduced MD 6 compression function $f_{Q}^{\pi}$ defined by equation (1) is $\left(t, q_{F}, q_{S}, \epsilon\right)$-indifferentiable from a random oracle $F$, for any number of queries $q_{F}$ and $q_{S}$, for distinguishing advantage

$$
\epsilon=\frac{\left(q_{S}+q_{F}\right)^{2}}{2^{n w}}+\frac{q_{S}}{2^{q w}}+\frac{q_{S} q_{F}}{2^{(n-c) w}},
$$

and for running time of the simulator $t=O\left(q_{S} n w\right)$.

Proof. We use the approach of Coron et al. [9], who showed that the indifferentiability framework can be successfully applied to the analysis of hash functions built from simpler primitives (such as block ciphers or compression functions). We note that related results have been obtained by Bertoni et al. 5] in their proof of indifferentiability of "sponge functions."

In our case, because $\pi$ is a permutation, the oracle $G$ contains both $\pi$ and $\pi^{-1}$, and we need to simulate them both. Slightly abusing notation, we will write $S$ for the simulator of $\pi$ and $S^{-1}$ for the simulator of $\pi^{-1}$. Thus, we need to construct simulator programs $S$ and $S^{-1}$ for $\pi$ and $\pi^{-1}$ such that no distinguisher $D$ can distinguish (except with negligible probability) between the following two scenarios:

(A) The distinguisher has oracle access to $f_{Q}^{\pi}$, to $\pi$, and to $\pi^{-1}$.

(B) The distinguisher has oracle access to $F, S$, and $S^{-1}$.

We define the simulators $S, S^{-1}$ for $\pi, \pi^{-1}$ as follows:

1. $S$ and $S^{-1}$ always act consistently with each other and with previous calls, if possible. If not possible (i.e., there are multiple answers for a given query), they abort. 
2. To evaluate $S(X)$ where $X=Q \| x$, compute $y=F(x)$, then return $R \| y$ where $R$ is chosen randomly from in $\mathbf{W}^{n-c}$.

3. To evaluate $S(X)$ where $X$ does not start with $Q$, return a value $R$ chosen randomly from $\mathbf{W}^{n}$.

4. To evaluate $S^{-1}(Y)$ : return a random $N$ in $\mathbf{W}^{n}$ which does not start with $Q$ (i.e., from $\left.\mathbf{W}^{n} \backslash\left(Q \| \mathbf{W}^{n-q}\right)\right)$.

The running time of the simulators is at most $t=O\left(q_{S} n w\right)$. Next, we argue the indifferentiability of our construction. To this end, consider any distinguisher $D$ making at most $q_{S}$ to queries to $S / \pi$ and $S^{-1} / \pi^{-1}$ and at most $q_{F}$ queries to $F / f_{Q}^{\pi}$. To analyze the advantage of this distinguisher, we consider several games $G_{0}, G_{1}, \ldots, G_{7}$. For each game $G_{i}$ below, let $p_{i}=\operatorname{Pr}\left(D\right.$ outputs 1 in $\left.G_{i}\right)$. Intuitively, $G_{0}$ will be the "real" game, $G_{7}$ will be the "ideal" game, and the intermediate game will slowly transform these games into each other.

Game $G_{0}$. This is the interaction of $D$ with $f_{Q}^{\pi}, \pi, \pi^{-1}$.

Game $G_{1}$. The game is identical to $G_{0}$ except the permutation $\pi$ is chosen in a "lazy" manner. Namely, we introduce a controller $C_{\pi}$ which maintains a table $T_{\pi}$ consisting of all currently defined values $(X, Y)$ such that $\pi(X)=Y$. Initially, this table is empty. Then, whenever a value $\pi(X)$ or $\pi^{-1}(Y)$ is needed, $C_{\pi}$ first checks in $T_{\pi}$ whether the corresponding value is already defined. If yes, it supplies it consistently. Else, it chooses the corresponding value at random subject to the "permutation constraint". Namely, if $T_{\pi}=\left\{\left(X_{i}, Y_{i}\right)\right\}$, then $\pi(X)$ is drawn uniformly from $\mathbf{W}^{n} \backslash\left\{Y_{i}\right\}$ and $\pi^{-1}(Y)$ is drawn uniformly from $\mathbf{W}^{n} \backslash\left\{X_{i}\right\}$. It is clear that $G_{1}$ is simply a syntactic rewriting of $G_{0}$. Thus, $p_{1}=p_{0}$.

Game $G_{2}$. This game is identical to $G_{1}$ except the controller $C_{\pi}$ does not make an effort to respect the permutation constraint above. Instead, it simply chooses undefined values $\pi(X)$ and $\pi^{-1}(Y)$ completely at random from $\mathbf{W}^{n}$, but explicitly aborts the game in case the permutation constraint is not satisfied. It is clear that $\left|p_{2}-p_{1}\right|$ is at most the probability of such an abort, which, in turn, is at most $\left(q_{S}+q_{F}\right)^{2} / 2^{n w}$.

Game $G_{3}$. This game is identical to $G_{2}$ except the controller $C_{\pi}$ does not choose values starting with $Q$ when answering the new inverse queries $\pi^{-1}(Y)$. Namely, instead of choosing such queries at random from $\mathbf{W}^{n}$, it chooses them at random from $\mathbf{W}^{n} \backslash\left(Q \| \mathbf{W}^{n-q}\right)$. It is easy to see that $\left|p_{3}-p_{2}\right|$ is at most the probability that $C_{\pi}$ would choose an inverse starting with $Q$ in the game $G_{2}$, which is at most $q_{S} / 2^{q w}$.

Game $G_{4}$. This game is identical to $G_{3}$ except we modify the controller $C_{\pi}$ as follows. Notice that there are three possible ways in which $C_{\pi}$ would add an extra entry to the table $T_{\pi}$ :

1. $D$ makes a query $\pi(X)$ to $\pi$, in which case a new value $(X, Y)$ might be added (for random $Y$ ). We call such additions forward. 
2. $D$ makes a query $\pi^{-1}(Y)$ to $\pi^{-1}$, in which case a new value $(X, Y)$ is added (for random $X$ not starting with $Q$ ). We call such additions backward.

3. $D$ makes a query $f_{Q}^{\pi}(x)=\chi_{c w}(\pi(Q \| x))$, in which case $C_{\pi}$ needs to evaluate $\pi(Q \| x)$ and add a value $(Q \| x, Y)$ (for random $Y$ ). We call such additions forced.

We start by making a syntactic change. When a forced addition $(Q \| x, Y)$ to $T_{\pi}$ is made, $C_{\pi}$ will mark it with a special symbol and will call this entry marked. $C_{\pi}$ will keep it marked until $D$ asks the usual forward query to $\pi(Q \| x)$, in which case the entry will become unmarked, just like all the regular forward and backward additions to $T_{\pi}$. With this syntactic addition, we can now make a key semantic change in the behavior of the controller $C_{\pi}$.

- In game $G_{3}$, when a backward query $\pi^{-1}(Y)$ is made, $C_{\pi}$ scans the entire table $T_{\pi}$ to see if an entry of the form $(X, Y)$ is present. In the new game $G_{4}, C_{\pi}$ will only scan the unmarked entries in $T_{\pi}$, completely ignoring the currently marked entries.

We can see that the only way the distinguisher $D$ will notice a difference between $G_{3}$ and $G_{4}$ is if $D$ can produce a backward query $\pi^{-1}(Y)$ such that the current table $T_{\pi}$ contains a marked entry of the form $(Q \| x, Y)$. Let us call this event $E$, and let us upper-bound the probability of $E$. For each forced addition $(Q \| x, Y)$, the value $Y$ is chosen at random from $\mathbf{W}^{n}$, and the distinguisher $D$ only learns the "chopped" value $y=\chi_{c w}(Y)$. In other words, $D$ does not see $(n-c) w$ completely random bits of $Y$. Thus, for any particular forced addition, the probability that $D$ ever "guesses" these missing bits is $2^{-(n-c) w}$. Since $D$ gets at most $q_{S}$ attempts, and there are at most $q_{F}$ forced values to guess, we get that $\operatorname{Pr}(E) \leq q_{S} q_{F} / 2^{(n-c) w}$. Thus, $\left|p_{4}-p_{3}\right| \leq \frac{q_{S} q_{F}}{2^{(n-c) w}}$.

Game $G_{5}$. We introduce a new controller $C_{F}$, which is simply imitating a random function $F: \mathbf{W}^{n-q} \rightarrow \mathbf{W}^{c}$. Namely, $C_{F}$ keeps a table $T_{F}$, initially empty. When a query $x$ is made, $C_{F}$ checks if there is an entry $(x, y)$ in $T_{F}$. If so, it outputs $y$. Else, it picks $y$ at random from $\mathbf{W}^{c}$, adds $(x, y)$ to $T_{F}$, and outputs $y$. Now, we modify the behaviors of the controller $C_{\pi}$ for $\pi / \pi^{-1}$ from the game $G_{4}$ as follows. In game $G_{4}$, when a new forward query $(Q \| x)$ was made to $\pi$, or a new query $x$ was made to $f_{Q}^{\pi}, C_{\pi}$ chose a random $Y$ from $\mathbf{W}^{n}$ and set $\pi(Q \| x)=Y$. In game $G_{5}$, in either one of these cases, $C_{\pi}$ will send a query $x$ to the controller $C_{F}$, get the answer $y$, and then set $Y=R \| y$, where $R$ is chosen at random from $\mathbf{W}^{n-c}$.

We notice that the game $G_{5}$ is simply a syntactic rewriting of the game $G_{4}$, since choosing a random value in $\mathbf{W}^{n}$ is equivalent to concatenating two random values in $\mathbf{W}^{n-c}$ and $\mathbf{W}^{c}$. Thus, $p_{5}=p_{4}$.

Game $G_{6}$. Before describing this game, we make the following observations about the game $G_{5}$. First, we claim that all the entries of the form $(Q \| x, Y)$ in $T_{\pi}$, whether marked or unmarked, have come from the explicit interaction with the controller $C_{F}$. Indeed, because in game $G_{3}$ we restricted $C_{\pi}$ to never 
answer a backward query so that the answer starts with $Q$, all such entries in $T$ have come either from a forward query $\pi(Q \| x)$, or the $f_{Q}^{\pi}$-query $f_{Q}^{\pi}(x)$. In either case, in game $G_{5}$ the controller $C_{\pi}$ "consulted" $C_{F}$ before making the answer. In fact, we can say more about the $f_{Q}^{\pi}$-query $f_{Q}^{\pi}(x)$. The answer to this query was simply the value $y$ which $C_{F}$ returned to $C_{\pi}$ on input $x$. Moreover, because of the rules introduced in game $G_{4}, C_{\pi}$ immediately marked the entry $(Q\|x, R\| y)$ which it added to $T_{\pi}$, and completely ignored this entry when answering the future backward queries to $\pi^{-1}$ (until a query $\pi(Q \| x)$ to $\pi$ was made).

Thus, we will make the following change in the new game $G_{6}$. When $D$ asks a new query $f_{Q}^{\pi}(x)$, the value $x$ no longer goes to $C_{\pi}$ (which would then attempt to define $\pi(Q \| x)$ by consulting $\left.C_{F}\right)$. Instead, this query goes directly to $C_{F}$, and $D$ is given the answer $y$. In particular, $C_{\pi}$ will no longer need to mark any of the entries in $T_{\pi}$, since all the $f_{Q}^{\pi}$ queries are now handled directly by $C_{F}$. More precisely, $C_{\pi}$ will only "directly" define the forward queries $\pi(X)$ and the backward queries $\pi^{-1}(Y)$ (in the same way it did in Game $G_{5}$ ), but no longer define $\pi(Q \| x)$ as a result of $D$ 's call to $f_{Q}^{\pi}(x)$.

We claim that game $G_{6}$ is, once again, only a syntactic rewriting of game $G_{5}$. Indeed, the only change between the two games is that, in game $G_{5}, T_{\pi}$ will contain some marked entries $(Q\|x, R\| y)$, which will be ignored anyway in answering all the inverse queries, while in Game $G_{6}$ such entries will be simply absent. There is only one very minor subtlety. In Game $G_{5}$, if $D$ first asks $f_{Q}^{\pi}(x)$, and later asks $\pi(Q \| x)$, the latter answer $R \| y$ will already be stored in $T_{\pi}$ at the time of the first question $f_{Q}^{\pi}(x)$. However, it will be marked and ignored until the second question $\pi(Q \| x)$ is made. In contrast, in Game $G_{6}$ this answer will only be stored in $T_{\pi}$ after the second question. However, since in both cases $C_{\pi}$ would answer by choosing a random $R$ and concatenating it with $C_{F}$ 's answer $y$ to $x$, this minor difference results in the same view for $D$. To sum up, $p_{6}=p_{5}$.

Game $G_{7}$. This is our "ideal" game where $D$ interacts with $S / S^{-1}$ and a true random oracle $F$. We claim this interaction is identical to the one in Game $G_{6}$. Indeed, $C_{F}$ is simply a "lazy" evaluation of the random oracle $F$. Also, after all our changes, the controller $C_{\pi}$ in Game $G_{6}$ is precisely equivalent to our simulators $S$ and $S^{-1}$. Thus, $p_{7}=p_{6}$.

Collecting all the pieces together, we get that the advantage of $D$ in distinguishing Game $G_{0}$ and Game $G_{7}$ is at most the claimed value

$$
\epsilon \leq \frac{\left(q_{S}+q_{F}\right)^{2}}{2^{n w}}+\frac{q_{S}}{2^{q w}}+\frac{q_{S} q_{F}}{2^{(n-c) w}}
$$

(In practice, there are other inputs to consider, such as the key input $K$, the unique ID $U$, and the control word $V$. The above proof applies as given, assuming that these inputs are available for the distinguisher to control. This is the correct assumption to make from the viewpoint of the MD6 mode of operation or other applications using the MD6 compression function.) 
Remark 1. We remark that our indifferentiability proof for the function $f_{Q}^{\pi}(x)=$ $\chi_{c w}(\pi(Q \| x))$ trivially generalizes to any compression function $f^{\pi}(x)$ of the form $f^{\pi}(x)=h_{c w}\left(\pi\left(g_{q w}(x)\right)\right)$, where:

$-h_{c w}: \mathbf{W}^{n} \rightarrow \mathbf{W}^{c}$ is any regular ${ }^{2}$ function. For the case of MD6, we use the "chop function" $h_{c w}=\chi_{c w}$.

$-g_{q w}: \mathbf{W}^{n-q} \rightarrow \mathbf{W}^{n}$ is any injective function which is (a) efficiently invertible and (b) efficiently verifiable (i.e., one can determine whether or not a point $y \in \mathbf{W}^{n}$ belongs to the range of $\left.g_{q w}\right)$. For the case of MD6, we use the "prepend function" $g_{q w}(x)=(Q \| x)$, for some constant $Q \in \mathbf{W}^{q}$.

\section{Indifferentiability of Tree-Based Modes of Operation}

In this section, we prove that any tree-based mode of operation, with certain properties (defined below), is indifferentiable from a random oracle when the compression function is a random oracle. (In fact, our result applies to modes of operation that can be described as straight-line programs, which are more general than trees.) We then derive the indifferentiability of the MD6 mode of operation as a consequence of this result.

Consider a compression function $\phi: \mathbf{W}^{\eta} \rightarrow \mathbf{W}^{c}$. Let $\mu^{\phi}:\{0,1\}^{*} \rightarrow\{0,1\}^{d}$ denote the mode of operation $\mu$ applied to $\phi$. We will prove that, if $\phi$ is a random oracle and $\mu$ satisfies the required properties, then $\mu^{\phi}$ is indifferentiable from a random oracle.

\subsection{Required Properties of Mode of Operation}

To prove indifferentiability of a mode of operation $\mu$, we will require $\mu$ to have the following properties. These are properties of the mode $\mu$ itself, independent of the particular compression function $\phi$.

Unique Parsing. Every compression function input $x \in \mathbf{W}^{\eta}$ that occurs in the computation of $\mu^{\phi}(M)$ for some $M \in\{0,1\}^{*}$ must be efficiently and uniquely parsable into a sequence of blocks (not necessarily of the same size), each of which is a compression function output, raw message bits (from the message being hashed), or metadata. Note that there may be parsable inputs that do not actually occur in the computation of $\mu^{\phi}(M)$ for any $M$.

Parent Predicate. Given the unique parsing property, we define a predicate parent $(x, y, i)$ which, given oracle access to a compression function $\phi$, takes two inputs $x, y \in \mathbf{W}^{\eta}$, and an index $i$. It outputs true iff $\phi(y)$ is equal to the $i$ th compression function output in the parsing of $x$. We say that $x$ is a parent of $y$, or equivalently, $y$ is a child of $x$ if $\operatorname{parent}(x, y, i)$ is true for some $i$. We also say that $y$ is the $i$ th child of $x$ if $\operatorname{parent}(x, y, i)$ is true. Note that actual parent-child pairs occurring during the execution of $\mu$ may satisfy

${ }^{2}$ A function is regular if every value in the range has an equal number of preimages in the domain. 
additional conditions: for example, $x$ and $y$ may contain consecutive level numbers. Our definition of parent does not verify these conditions, and thus may create a parent relationship where none should exist. These conditions (among others) will be verified by a function $\rho$ defined below.

Leaf Predicate. Given the unique parsing property, we also define a predicate leaf $(x)$ on compression function inputs $x$ which returns true iff the parsing of $x$ contains only message bits and metadata but no compression function outputs. We say that $x$ is a leaf if leaf $(x)$ is true.

Root Predicate. There must be a well-defined, efficiently testable predicate $\operatorname{root}(x)$ such that: for any $M \in\{0,1\}^{*}$, for every non-final compression function call $x$ in the computation of $\mu^{\phi}(M), \operatorname{root}(x)$ is false, and for the final compression function call $y, \operatorname{root}(y)$ is true. For strings $x$ that are neither non-final nor final compression function calls in the computation of $\mu^{\phi}(M)$ for any $M, \operatorname{root}(x)$ can be either true or false.

Note that this condition implies that the set of strings $x \in \mathbf{W}^{\eta}$ that are the final compression call for some $M$ must be disjoint from the set of strings $y \in \mathbf{W}^{\eta}$ that are a non-final compression call for some $M$.

Straight-Line Program Structure. The mode of operation $\mu$ must be a straight-line program in the following sense. It carries out a sequence of calls to the compression function, where input to call number $i$ in the sequence is computed from the message itself, metadata, and outputs of some $j$ calls numbered $i_{1}, i_{2}, \ldots, i_{j}<i$. This sequence (that is, the exact dependence of $i$ th call on the previous calls and the message) must be deterministically computable from $M$ alone, regardless of $\phi$. For every call in the sequence except the last one, its output value must be used to compute some other input value. Moreover, for any $\phi$, the output values of calls $i_{1}, i_{2}, \ldots, i_{j}$ must occur in the parsing of the input to the $i$ th call. The last call $x$ in that sequence must have $\operatorname{root}(x)=$ true; for all the others, the root predicate must be false. Denote by $\Sigma(M)$ the set of all calls to $\phi$ (input and output pairs) during the computation of $\mu^{\phi}(M)$.

Final Output Processing. It must be the case that $\mu^{\phi}(M)=\zeta(\phi(x))$ where $x$ is the final compression input, where $\zeta: \mathbf{W}^{c} \rightarrow\{0,1\}^{d}$ is an efficiently computable, regular function. The set of all preimages $\zeta^{-1}(h)$ of a value $h$ must be efficiently sampleable given $h$.

Message Reconstruction. There must be an efficiently computable function $\rho$ that takes a set $\Pi$ of compression function calls and returns a message $M$ if $\Pi=\Sigma(M)$, and $\perp$ otherwise. Because $\mu$ is deterministic, it follows that if $\rho\left(\Pi_{1}\right)=M_{1}$ and $\rho\left(\Pi_{2}\right)=M_{2}$ and $\Pi_{1} \neq \Pi_{2}$, then $M_{1} \neq M_{2}$.

We let $\kappa(\ell)$ denote an upper bound on the running time of $\rho$ on an input set $\Pi$ containing at most $\ell$ compression function calls. We assume that the other efficiently computable operations defined above (compression function input parsing, computing $\zeta(C)$, sampling from $\zeta^{-1}(h)$, and evaluating root $\left.(x)\right)$ run in constant time. 


\subsection{The Simulator}

For the proof of indifferentiability of $\mu$, we will define a polynomial-time simulator $S_{0}$ for the compression function $\phi . S_{0}$ works as follows.

$S_{0}$ maintains a set $T$, initially empty, of pairs $(x, C) \in \mathbf{W}^{\eta} \times \mathbf{W}^{c}$ such that it has responded with $C$ to a query $x$.

Upon receiving a compression function query $x^{*}, S_{0}$ searches its set $T$ for a pair $\left(x^{*}, C^{*}\right)$. If it finds such a pair, $S_{0}$ returns $C^{*}$.

Otherwise, $S_{0}$ evaluates $\operatorname{root}\left(x^{*}\right)$. If false, $S_{0}$ chooses a fresh random string $C^{*} \in \mathbf{W}^{c}$, inserts $\left(x^{*}, C^{*}\right)$ into $T$, and returns $C^{*}$.

If true, $S_{0}$ executes the following "reconstruction procedure" to determine whether $x^{*}$ is the final compression function call in a computation of $\mu^{\phi}(M)$, all of whose non-final compression function calls have already been seen by $S_{0}$. In the reconstruction procedure, $S_{0}$ will build a set $\Pi$, initially empty, of pairs $(x, C)$ of compression function inputs and outputs.

1. Parse $x^{*}$ into an ordered sequence of message bits, metadata, and compression function outputs. Let $j$ be the number of compression function outputs.

2. For $i=1, \ldots, j$ :

For each pair $(x, C)$ in $T$, evaluate $\operatorname{parent}\left(x^{*}, x, i\right)$. If no pair $(x, C)$ satisfies $\operatorname{parent}\left(x^{*}, x, i\right)$, or if multiple pairs $(x, C)$ satisfy $\operatorname{parent}\left(x^{*}, x, i\right)$, quit the reconstruction procedure; choose a fresh random string $C^{*} \in \mathbf{W}^{c}$, insert $\left(x^{*}, C^{*}\right)$ into $T$, and return $C^{*}$. If a unique child pair $(x, C)$ is found, add $(x, C)$ to $\Pi$. If leaf $(x)$ is false, execute steps 1 and 2 for $x$.

If $S_{0}$ completes the reconstruction procedure (without returning a fresh random string), $S_{0}$ now calls $\rho(\Pi)$. If $\rho$ returns $\perp, S_{0}$ chooses a fresh random string $C^{*} \in \mathbf{W}^{c}$, inserts $\left(x^{*}, C^{*}\right)$ into $T$, and returns $C^{*}$. If $\rho$ returns a message $M^{*}$, $S_{0}$ calls $F$ on $M^{*}$ and samples a string $C^{*} \in \mathbf{W}^{c}$ randomly from $\zeta^{-1}\left(F\left(M^{*}\right)\right)$. $S_{0}$ inserts $\left(x^{*}, C^{*}\right)$ into $T$ and returns $C^{*}$.

Running Time. Let $q_{t}$ be the total number of compression function queries to the simulator. To answer each compression function query, the $S_{0}$ takes $O\left(q_{t}^{2}+\kappa\left(q_{t}\right)\right)$ time. Therefore, the total running time of the simulator is $O\left(q_{t}^{3}+q_{t} \cdot \kappa\left(q_{t}\right)\right)$.

Correctness. In our proof, we will use the following correctness properties of the simulator.

Property 1: Suppose that there are no collisions on the output of $S_{0}$ (equivalently, there are no Type 1 events, which we define later). If all of the non-final compression calls in the computation of $\mu^{\phi}\left(M^{*}\right)$ have been made to $S_{0}$, then on the final compression call $x^{*}, S_{0}$ will reconstruct $M^{*}$ and consult $F$ on $M^{*}$. This property can be easily proven by induction, starting at the final query and working backward; the straight-line program property ensures that all the compression function calls in $\Sigma\left(M^{*}\right)$ will be found, and the assumption of no collisions ensures that no extra calls will be found.

Property 2: Suppose $S_{0}$ reconstructs $M$ in response to a query at time $\tau$. Then all of the compression calls in the computation of the $\mu^{\phi}(M)$ have been made to $S_{0}$ by time $\tau$ (indeed, otherwise $\rho(\Pi)$ would not return $M$, by definition). 


\subsection{Games}

To prove the indifferentiability of the mode of operation $\mu$, we consider a distinguisher $D$ making compression function queries and mode of operation queries. We define a sequence of games, $G_{0}$ through $G_{4}$. Game $G_{0}$ is the "ideal" game, where $D$ has oracle access to the random oracle $F$ and the simulator $S_{0}$. Game $G_{4}$ is the "real" game, where $D$ has oracle access to $\mu^{\phi}$ and the random oracle $\phi$.

For each game $G_{i}$, let $p_{i}$ denote the probability that $D$ outputs 1 in game $G_{i}$. We will argue that the view of the distinguisher cannot differ between consecutive games with more than negligible probability.

The games are defined as follows.

Game $G_{0}$. In Game $G_{0}$, the distinguisher $D$ interacts with the random oracle $F$ and the polynomial-time simulator $S_{0}$ defined above.

Game $G_{1}$. In Game $G_{1}$, we modify the simulator. At the start of the game, the new simulator $S_{1}$ uses its random coins to specify a random oracle $\mathcal{O}_{S}$ : $\mathbf{W}^{\eta} \rightarrow \mathbf{W}^{c}$. The subsequent behavior of $S_{1}$ is identical to that of $S_{0}$ except, whereas $S_{0}$ generates its random bits in a lazy manner, $S_{1}$ gets its random bits from $\mathcal{O}_{S}$. Specifically, wherever $S_{0}$ answers a query $x^{*}$ with a fresh random string $C^{*} \in \mathbf{W}^{c}, S_{1}$ answers with $\mathcal{O}_{S}\left(x^{*}\right)$. Similarly, wherever $S_{0}$ answers a query $x^{*}$ by sampling randomly from $\zeta^{-1}\left(F\left(M^{*}\right)\right), S_{1}$ samples randomly from $\zeta^{-1}\left(F\left(M^{*}\right)\right)$ using $\mathcal{O}_{S}\left(x^{*}\right)$ as its source of random bits.

The view of the distinguisher is the same in Game $G_{1}$ and Game $G_{0}$, so we have $p_{1}=p_{0}$.

Game $G_{2}$. In Game $G_{2}$, we introduce a relay algorithm $R_{0}$ between $D$ and $F$. The relay algorithm $R_{0}$ has oracle access to $F$ and simply relays $D$ 's mode of operation queries to $F$ and relays $F$ 's responses back to $D$.

The view of the distinguisher is the same in Game $G_{2}$ and Game $G_{1}$, so we have $p_{2}=p_{1}$.

Game $G_{3}$. In Game $G_{3}$, we modify the relay algorithm. Instead of querying $F$, the new relay algorithm $R_{1}$ computes the mode of operation on its input, querying $S_{1}$ on each compression function call.

Game $G_{4}$. This is the final game. In Game $G_{4}$, we modify the simulator so that it no longer consults $F$. The new simulator $S_{2}$ always responds to a new query $x^{*}$ with $\mathcal{O}_{S}\left(x^{*}\right)$. Thus, in this game $D$ interacts with the mode of operation $\mu^{\mathcal{O}_{S}}$ and the random oracle $\mathcal{O}_{S}$.

Bad Events. In order to argue that the view of $D$ cannot differ between Game $G_{2}$ and Game $G_{3}$ with more than negligible probability, we first define three types of "bad events" that can occur in Game $G_{2}$ or Game $G_{3}$.

- Type 1: $S_{1}$ inserts a pair $\left(x_{2}, C\right)$ into $T$ when there is already a pair $\left(x_{1}, C\right)$ such that $x_{1} \neq x_{2}$. 
- Type 2: $S_{1}$ inserts a pair $\left(x_{2}, C_{2}\right)$ into $T$ when there is already a pair $\left(x_{1}, C_{1}\right)$ in $T$ such that $x_{1}$ is a parent of $x_{2}$.

- Type 3: $D$ makes a query $x_{2}$ to $S_{1}$ such that $x_{2}$ is a parent of an $x_{1}$ which was previously queried by $R$ but never directly by $D$.

We now prove that if none of these bad events occur in Game $G_{3}$, then the view of $D$ is identical in $G_{2}$ and $G_{3}$.

Lemma 1. For fixed coins of $F, S_{1}$, and $D$, if no bad events occur in Game $G_{3}$, then the view of $D$ is identical in Game $G_{2}$ and Game $G_{3}$.

Proof. We fix the random coins of $F, S_{1}$, and $D$ and assume no bad events occur. We show by induction that $D$ 's observable values (the responses of the relay algorithm and the simulator) are identical in Games $G_{2}$ and $G_{3}$.

Suppose that the observable values have been identical in Games $G_{2}$ and $G_{3}$ so far. Consider $D$ 's next query. It is either a mode of operation query or a compression function query.

Mode of Operation Query. Consider a query $M^{*}$ to $\mu^{\phi}$. In Game $G_{2}, R_{0}$ always returns $F\left(M^{*}\right)$. In Game $G_{3}, R_{1}$ will return $F\left(M^{*}\right)$ if the response of $S_{1}$ on the final compression call $x^{*}$ is sampled from $\zeta^{-1}\left(F\left(M^{*}\right)\right)$. There are two cases two consider.

1. $x^{*}$ is a new query to $S_{1}$. Since $R_{1}$ has made all of the non-final calls for $M^{*}$ before $x^{*}$, assuming there are no Type 1 events, by Correctness Property 1 of the simulator, $S_{1}$ will reconstruct $M^{*}$ and return a string sampled from $\zeta^{-1}\left(F\left(M^{*}\right)\right)$

2. $x^{*}$ has already been queried to $S_{1}$ before. Suppose it was first queried at time $\tau$. Consider $x^{*}$ 's children in the current computation $\mu^{\phi}(x)$. All of these children must have also been seen before time $\tau$. Otherwise, a Type 2 event occurred in Game $G_{3}$ during one of the calls by $R_{1}$ after time $\tau$. By induction, all of $R_{1}$ 's calls in the computation of $\mu^{\phi}(x)$ must have been seen before time $\tau$. Therefore, assuming there are no Type 1 events, by Correctness Property 1 of the simulator, $S_{1}$ reconstructed $M^{*}$ and returned a string sampled from $\zeta^{-1}\left(F\left(M^{)}\right)\right.$at time $\tau$.

Therefore, given that the observables have been the same so far, if the next query is a mode of operation query, the next observable will be the same in games $G_{2}$ and $G_{3}$.

Compression Function Query. Consider a query $x^{*}$ to $\phi$.

We first make the following observation. Let $T_{2}$ be the set of queries $S_{1}$ has seen so far in Game $G_{2}$. Let $T_{3}$ be the set of queries $S_{1}$ has seen so far in Game $G_{3}$. Assuming the observables in the two games have been identical so far, $T_{2}$ must be a subset of $T_{3}$. This is because $T_{2}$ contains only the compression function queries made by $D$ and $T_{3}$ contains these queries along with the queries made by $R_{1}$ in computing responses to $D$ 's mode of operation queries. 
Now, suppose that $x^{*}$ has already previously been queried to $S_{1}$ in Game $G_{2}$. Then $S_{1}$ 's response will be the same in both games, since by assumption it was the same in both games the first time $x^{*}$ was queried.

So suppose that $x^{*}$ is a new query in Game $G_{2}$. If in Game $G_{2}, S_{1}$ reconstructs an $M^{*}$ and returns an element of $\zeta^{-1}\left(F\left(M^{*}\right)\right)$ sampled using $\mathcal{O}_{S}\left(x^{*}\right)$ for its random bits, then by Correctness Property 2 of the simulator, all of $M^{*}$ 's queries are in $T_{2}$, and therefore all of $M^{*}$ 's queries are in $T_{3}$. Therefore, assuming no Type 1 events, by Correctness Property $1, S_{1}$ must also reconstruct $M^{*}$ in Game $G_{3}$ and return an element of $\zeta^{-1}\left(F\left(M^{*}\right)\right)$ sampled using $\mathcal{O}_{S}\left(x^{*}\right)$ for its random bits.

If in Game $G_{2}, S_{1}$ instead returns $\mathcal{O}_{S}\left(x^{*}\right)$, then $S_{1}$ 's answer in Game $G_{3}$ is the same unless in Game $G_{3} S_{1}$ reconstructs an $M^{*}$. If $S_{1}$ reconstructs an $M^{*}$ in Game $G_{3}$ but not in Game $G_{2}$, then at least one of the queries used in the reconstruction must have come from $R_{1}$. But $D$ made a query for the final compression function input $x^{*}$. Consider all of $x^{*}$ 's children in $G_{3}$. All of these queries must have been asked by $D$ at some time. Otherwise, a Type 3 event occurred in game $G_{3}$. By induction, all of the queries for $M^{*}$ must have been asked by $D$ before $x^{*}$, but then (assuming no Type 1 events) $S_{1}$ would have reconstructed $M^{*}$ in Game $G_{2}$, contradicting the assumption that $S_{1}$ returns $\mathcal{O}_{S}\left(x^{*}\right)$ in Game $G_{2}$.

Therefore, given that the observables have been the same so far, if the next query is a mode of operation query, the next observable will be the same in games $G_{2}$ and $G_{3}$.

Therefore, conditioned on there being no occurrences of bad events in $G_{3}$, the view of $D$ is identical in games $G_{2}$ and $G_{3}$.

We now bound the probability of bad events in Game $G_{3}$.

Lemma 2. Suppose $D$ makes $q_{S}$ compression function queries and generates $q_{t}$ compression function calls from its mode of operation queries and its compression function queries. Let $\operatorname{Pr}[\mathrm{Bad}]$ denote the probability that a bad event occurs in Game $G_{3} . \operatorname{Pr}[\mathrm{Bad}] \leq(2 \eta / c+1) q_{t}^{2} / 2^{c w}$.

Proof. We first make the observation that on a new query $x, S_{1}$ always responds with a fresh random string in $\mathbf{W}^{c}$. This is because $S_{1}$ responds with either (a) $\mathcal{O}_{S}(x) \in \mathbf{W}^{c}$ or (b) a random sample from $\zeta^{-1}(F(M))$ using $\mathcal{O}_{S}(x)$ as its source of randomness. In case (a), $S_{1}$ 's response is a fresh random string in $\mathbf{W}^{c}$. In case (b), $S_{1}$ 's is a fresh random string in $\mathbf{W}^{c}$ as long as $S_{1}$ has not queried $F$ on $M$ before. Since a message $M$ has a unique final compression call and $x$ is a new query, $S_{1}$ cannot have queried $F$ on $M$ before.

We now consider the three types of bad events.

Type 1 event: A Type 1 event corresponds to a collision between two random $c$-word strings among at most $q_{t}$ compression function queries. We can bound the probability using the birthday bound over $q_{t}$ random $c$-word strings: $\operatorname{Pr}\left[\right.$ Type 1] $\leq q_{t}^{2} / 2^{c w+1}$.

Type 2 event: A Type 2 event occurs when $S_{1}$ 's random $c$-word response $C_{2}$ to a query $x_{2}$ equals one of the compression function outputs in the parsing of a 
previous query $x_{1}$. There at most $\eta / c$ compression function outputs in the parsing of any query. So, there are at most $q_{t} \cdot \eta / c$ compression function outputs for $S_{1}$ to "guess" and $S_{1}$ has at most $q_{t}$ guesses. Therefore, $\operatorname{Pr}[$ Type 2$] \leq\left(\eta / c \cdot q_{t}^{2}\right) / 2^{c w}$.

Type 3 event: A Type 3 event occurs when $D$ makes a query which is a parent of a query that $R$ made but $D$ did not. The only output that $D$ sees from $R$ 's queries is $\zeta(f(x))$ for the final compression call $x$, but it is not possible to make a query which is a parent of a final compression call. The probability of $D$ "guessing" a non-final compression function output that it has not seen is $1 / 2^{c w}$. There are at most $\left(q_{t}-q_{S}\right)$ outputs to guess and $D$ has at most $q_{S} \cdot \eta / c$ guesses. Therefore, $\operatorname{Pr}\left[\right.$ Type 3] $\leq\left(\eta / c \cdot q_{S}\left(q_{t}-q_{S}\right)\right) / 2^{c w}$.

Summing together, we get $\operatorname{Pr}[\mathrm{Bad}] \leq q_{t}^{2} / 2^{c w+1}+\left(\eta / c \cdot q_{t}^{2}\right) / 2^{c w}+\left(\eta / c \cdot q_{S}\left(q_{t}-\right.\right.$ $\left.\left.q_{S}\right)\right) / 2^{c w} \leq(2 \eta / c+1) q_{t}^{2} / 2^{c w}$.

\subsection{Indifferentiability Theorem}

We now state our theorem of indifferentiability of $\mu$.

Theorem 2. If $\phi: \mathbf{W}^{\eta} \rightarrow \mathbf{W}^{c}$ is a random oracle, then $\mu^{\phi}$ is $\left(t, q_{F}, q_{S}, \epsilon\right)$ indifferentiable from a random oracle $F:\{0,1\}^{*} \rightarrow\{0,1\}^{d}$, with $\epsilon=(2 \eta / c+$ 1) $q_{t}^{2} / 2^{c w}$ and $t=O\left(q_{t}^{3}+q_{t} \cdot \kappa\left(q_{t}\right)\right)$, for any $q_{F}$ and $q_{S}$ such that the total number of compression function calls from the mode of operation queries and the compression function queries of the distinguisher is at most $q_{t}$.

Proof. Consider a distinguisher $D$ that makes $q_{S}$ compression function queries and generates $q_{t}$ compression function calls from its mode of operation queries and its compression function queries.

It can easily be seen that the view of $D$ is the same in Games $G_{0}, G_{1}$, and $G_{2}$, so $p_{2}=p_{1}=p_{0}$.

Combining Lemma 1 and Lemma 2, we have that $\left|p_{3}-p_{2}\right| \leq(2 \eta / c+1) q_{t}^{2} / 2^{c w}$.

It is straightforward to see that $p_{4}=p_{3}$. To see this, consider a query $x^{*}$ (from either $D$ or $R_{1}$ ) to $S_{2}$. If $S_{2}$ has seen the query $x^{*}$ before, $S_{2}$ repeats the answer it gave the first time it was queried on $x^{*}$. If $x^{*}$ is a new query, then in $G_{4}, S_{2}$ responds with the fresh random string $\mathcal{O}_{S}\left(x^{*}\right)$. As argued previously in the proof of Lemma 2, $S_{1}$ always responds to a new query $x^{*}$ with a fresh random string in $\mathbf{W}^{c}$. Thus, the view of $D$ is unchanged from $G_{3}$ to $G_{4}$, and $p_{4}=p_{3}$.

Summing over all the games, the total advantage of $D$ in distinguishing between $G_{0}$ and $G_{4}$ is at most the claimed $(2 \eta / c+1) q_{t}^{2} / 2^{c w}$.

This completes the proof of the theorem.

It follows from our indifferentiability theorem that the MD6 mode of operation $\mathcal{M}^{f_{Q}}$ (specified in [17) is indifferentiable from a random oracle when $f_{Q}$ is a random oracle. This result is stated below.

Corollary 1. If $f_{Q}: \mathbf{W}^{n-q} \rightarrow \mathbf{W}^{c}$ is a random oracle, the MD6 mode of operation $\mathcal{M}^{f_{Q}}$ is $\left(t, q_{F}, q_{S}, \epsilon\right)$-indifferentiable from a random oracle $F:\{0,1\}^{*} \rightarrow$ $\{0,1\}^{d}$, with $\epsilon=9 q_{t}^{2} / 2^{c w}$ and $t=O\left(q_{t}^{3}\right)$, for any $q_{F}$ and $q_{S}$ such that the total number of compression function calls from the mode of operation queries and the compression function queries of the distinguisher is at most $q_{t}$. 
Proof. It can easily be seen that the MD6 mode of operation $\mathcal{M}$ satisfies the required properties. In particular, for MD6 $\zeta$ is the function $\chi_{d}$ which returns the last $d$ bits of its input. The root $(x)$ predicate corresponds to testing whether the $z$ bit of $x$ is 1 . It is straightforward to define a message reconstruction algorithm $\rho$ for MD6 that runs in time $\kappa\left(q_{t}\right)=O\left(q_{t}\right)$.

Remark 2. The distinguishing probability $\epsilon$ and simulator running time $t$ stated above follow directly from Theorem 2

However, for MD6 specifically, we can easily get a tighter bound on $\epsilon$ because each compression function input has a unique node identifier, consisting of a tree level and index. Therefore, for a given compression function input $y$, in any other compression function input $x$, there is at most one (not $\eta / c=4$ ) compression function output that could cause $y$ to be a child of $x$. Thus, in bounding the probability of bad events for MD6, we can replace all instances of $\eta / c$ with 1 and get $\epsilon=\operatorname{Pr}[\mathrm{Bad}] \leq 2 q_{t}^{2} / 2^{c w}$.

Similarly, the presence of unique node identifiers in MD6 gives us a tighter bound on the running time $t$ of the simulator. By maintaining its previously seen queries in sorted order (sorted by node identifier), the simulator can run in time $O\left(q_{t}^{2}\right)$ instead of $O\left(q_{t}^{3}\right)$.

\section{Conclusion}

We have shown that the (reduced) compression function $\chi_{c w}(\pi(Q \| x))$ of MD6 is indifferentiable from a fixed-input-length random oracle when $\pi$ is a random permutation. We have also shown that any tree-based mode of operation with certain properties is indifferentiable from a variable-input-length random oracle when applied to a compression function that is a fixed-input-length random oracle. As a consequence of this result, the MD6 mode of operation is indifferentiable from a random oracle. Combined, these results imply that the design of MD6 has no stuctural weaknesses, such as "extension attacks", and that MD6 can be plugged into any application proven secure assuming a variable-input-length random oracle to obtain a scheme secure in a (fixed-length) random permutation model.

\section{References}

1. Aumasson, J.-P., Meier, W.: Nonrandomness observed on a reduced version of the compression function with 18 rounds in about $2^{17}$ operations

2. Bellare, M., Ristenpart, T.: Multi-property-preserving hash domain extension and the EMD transform. In: Lai, X., Chen, K. (eds.) ASIACRYPT 2006. LNCS, vol. 4284, pp. 299-314. Springer, Heidelberg (2006)

3. Bellare, M., Rogaway, P.: Random oracles are practical: A paradigm for designing efficient protocols. In: ACM Conference on Computer and Communications Security, pp. 62-73 (1993)

4. Bertoni, G., Daemen, J., Peeters, M., Van Assche, G.: Sponge functions (May 2007), http://www.csrc.nist.gov/pki/HashWorkshop/PublicComments/2007May.html 
5. Bertoni, G., Daemen, J., Peeters, M., Van Assche, G.: On the indifferentiability of the sponge construction. In: Smart, N.P. (ed.) EUROCRYPT 2008. LNCS, vol. 4965, pp. 181-197. Springer, Heidelberg (2008)

6. Bertoni, G., Daemen, J., Peeters, M., Van Assche, G.: Sufficient conditions for sound tree hashing modes. In: Handschuh, H., Lucks, S., Preneel, B., Rogaway, P. (eds.) Symmetric Cryptography. Dagstuhl Seminar Proceedings (2009), http://www. dagstuhl.de/Materials/index.en.phtml?09031

7. Chabaud, F., Joux, A.: Differential collisions of SHA-0. In: Krawczyk, H. (ed.) CRYPTO 1998. LNCS, vol. 1462, pp. 56-71. Springer, Heidelberg (1998)

8. Chang, D., Lee, S., Nandi, M., Yung, M.: Indifferentiable security analysis of popular hash functions with prefix-free padding. In: Lai, X., Chen, K. (eds.) ASIACRYPT 2006. LNCS, vol. 4284, pp. 283-298. Springer, Heidelberg (2006)

9. Coron, J.-S., Dodis, Y., Malinaud, C., Puniya, P.: Merkle-Damgård revisited: How to construct a hash function. In: Shoup, V. (ed.) CRYPTO 2005. LNCS, vol. 3621, pp. 430-448. Springer, Heidelberg (2005)

10. Crutchfield, C.Y.: Security proofs for the MD6 hash function mode of operation. Master's thesis, MIT EECS Department (2008), http://groups.csail.mit.edu/cis/theses/crutchfield-masters-thesis. pdf

11. Dinur, I., Shamir, A.: Cube attack on a reduced version of the compression function with 15 rounds

12. Dodis, Y., Pietrzak, K., Puniya, P.: A new mode of operation for block ciphers and length-preserving MACs. In: Smart, N.P. (ed.) EUROCRYPT 2008. LNCS, vol. 4965, pp. 198-219. Springer, Heidelberg (2008)

13. Maurer, U., Renner, R., Holenstein, C.: Indifferentiability, impossibility results on reductions, and applications to the random oracle methodology. In: Naor, M. (ed.) TCC 2004. LNCS, vol. 2951, pp. 21-39. Springer, Heidelberg (2004)

14. National Institute of Standards and Technology. Announcing request for candidate algorithm nominations for a new cryptographic hash algorithm (SHA-3) family. Federal Register Notices, vol. 72(212), pp. 62212-62220 (November 2, 2007), http://csrc.nist.gov/groups/ST/hash/documents/FR_Notice_Nov07.pdf

15. National Institute of Standards and Technology. Announcing the development of new hash algorithm(s) for the revision of Federal Information Processing Standard (FIPS) 1802, secure hash standard. Federal Register Notices, vol. 72(14), pp. 28612863 (January 23, 2007), http://csrc.nist.gov/groups/ST/hash/documents/FR_Notice_Jan07.pdf

16. Rivest, R.L.: Slides from invited talk at Crypto 2008 (2008), http://group.csail.mit.edu/cis/md6/Rivest-TheMD6HashFunction.ppt

17. Rivest, R.L., Agre, B., Bailey, D.V., Crutchfield, C., Dodis, Y., Fleming, K.E., Khan, A., Krishnamurthy, J., Lin, Y., Reyzin, L., Shen, E., Sukha, J., Sutherland, D., Tromer, E., Yin, Y.L.: The MD6 hash function: A proposal to NIST for SHA-3 (2008), http://groups.csail .mit.edu/cis/md6/submitted-2008-10-27/ Supporting_Documentation/md6_report.pdf

18. Wang, X., Lai, X., Feng, D., Chen, H., Yu, X.: Cryptanalysis of the hash functions MD4 and RIPEMD. In: Cramer, R. (ed.) EUROCRYPT 2005. LNCS, vol. 3494, pp. 1-18. Springer, Heidelberg (2005)

19. Wang, X., Yin, Y.L., Yu, H.: Finding collisions in the full SHA-1. In: Shoup, V. (ed.) CRYPTO 2005. LNCS, vol. 3621, pp. 17-36. Springer, Heidelberg (2005) 
20. Wang, X., Yu, H.: How to Break MD5 and Other Hash Functions. In: Cramer, R. (ed.) EUROCRYPT 2005. LNCS, vol. 3494, pp. 19-35. Springer, Heidelberg (2005)

21. Wang, X., Yu, H., Yin, Y.L.: Efficient Collision Search Attacks on SHA-0. In: Shoup, V. (ed.) CRYPTO 2005. LNCS, vol. 3621, pp. 1-16. Springer, Heidelberg (2005)

22. $\mathrm{Yu}, \mathrm{H}$., Wang, X.: Multicollision attack on the compression functions of MD4 and 3-pass HAVAL. IACR ePrint Archive, Report 2007/085 (2007),

http://eprint.iacr.org/2007/085 